\title{
Perspectiva de Tiempo Futuro y su relevancia motivacional en distintos contextos educativos
}

\section{Future Time Perspective and its Motivational Relevance in Different Educational Contexts}

\author{
Dora Isabel Herrera Paredes \\ Pontificia Universidad Católica del Perú, Lima, Perú \\ ORCID: https://orcid.org/0000-0002-8327-9435
}

Recibido 31-05-19 Revisado 14-08-19 Aprobado 15-11-19 En línea 18-11-19

*Correspondencia

Email: diherrer@pucp.pe

\section{Citar como:}

Herrera, D. (2019). Perspectiva de Tiempo Futuro y su relevancia motivacional en distintos contextos educativos. Propósitos y Representaciones, 7(SPE), e348. doi: http://dx.doi.org/10.20511/pyr2019.v7nSPE.348 


\section{Resumen}

El presente artículo sistematiza e integra los hallazgos de investigaciones desarrolladas en Perú sobre Perspectiva de Tiempo Futuro (PTF) en contextos educativos. La PTF es una variable psicológica motivacional que permite explicar el comportamiento presente partiendo de metas establecidas en el futuro. El concepto demostró solidez teórica-empírica en distintas latitudes. Durante la segunda mitad del siglo XX, Nuttin y Lens (1985) aplicaron el concepto en diversos ámbitos, especialmente el educativo. Su propuesta consideró la condición cognitiva y motivacional de la variable reportándose su densidad, extensión y nivel de realismo. Se ha trabajado empíricamente con este supuesto hasta la actualidad, pero hace una década se amplió dicho concepto hacia una propuesta multidimensional conceptualizada como Orientación Futura (OF) la cual posee tres componentes: motivacional, cognitivo y comportamental (Seginer, 2009). En Perú, desde 1998 se implementó estudios longitudinales y transversales que corroboraron consistentemente que, en adolescentes y jóvenes de varias instituciones educativas, la PTF es corta, el nivel de realismo bajo y la aspiración por ser profesional constante. En los últimos años, se ha reorientado la medición resaltando la multidimensionalidad del concepto. Los recientes hallazgos muestran que la relación entre el bienestar y el componente motivacional de la OF tiende a ser positiva e intensa en el dominio de carrera profesional de los jóvenes evaluados. Es importante desarrollar esta línea de investigación porque el análisis de la motivación y OF juvenil brindará la pauta para futuras intervenciones a favor de su óptima inserción social y bienestar.

Palabras clave: Perspectiva de Tiempo Futuro; Motivación; Satisfacción con la vida.

\section{Summary}

This study aims at systematizing and integrating research findings about Future Time Perspective (FTP) in Peruvian educational contexts. FTP is a motivational psychological variable that allows explaining the present behavior, considering goals set in the future. The construct demonstrated its theoretical and empirical strength in different contexts. During the second half of the $\mathrm{XXt}^{\mathrm{h}}$ Century Nuttin and Lens (1985) applied the variable in different fields, especially the educational one. Their proposal considered the cognitive and motivational properties of FTP; its density, extension and level of realism was then reported. Empirical research has been conducted to date under this theoretical background; however, a decade ago this concept was redirected to a multidimensional proposal conceptualized as Future Orientation (FO) considering three components: motivational, cognitive and behavioral (Seginer, 2009).In Peru, longitudinal and cross-sectional studies were carried out since 1998. These studies consistently documented that adolescents and youngsters studying in different educational institutions, showed a short FTP, a low level of realism and a keen aspiration for becoming professionals. During the last years, FO measuring was reoriented considering the multidimensional feature of the concept. Recent research findings in Peruvian adolescents and youngsters indicate that there is a positive and intense relation between satisfaction with life and the motivational component of FO. Taking into account these results, researchers consider that it is important to develop this line of investigation, as the analysis of the youngster's motivation and FO will provide the guidelines for future social interventions in favor of their social insertion and well-being.

Keywords: Future Time Perspective; Motivation; Satisfaction with Life.

\section{Introducción}

Tomando en consideración que la ciencia psicológica se orienta, desde sus orígenes, a describir, explicar y predecir el comportamiento humano y favorecer, a partir de sus hallazgos, el bienestar de las personas resulta claro que el estudio de la motivación ha ocupado un lugar destacado; pues a través de esta variable psicológica se puede comprender por qué los seres humanos realizan actividades distintas (Eccles \& Wigfield, 2002; Herrera \& Matos, 2009; Reeve, 2005). Diversas orientaciones teóricas se han aproximado al estudio de la motivación; no obstante, en el presente 
artículo, con el objetivo de presentar la síntesis de los hallazgos reportados sobre PTF, solo se considerará la relevancia motivacional de la Perspectiva de Tiempo Futuro que al ser analizada en distintos contextos educativos ha permitido desarrollar una línea de investigación a nivel internacional y particularmente en el Perú. Por ello, se sustentará en una primera sección sus aportes teóricos, luego se precisará su evolución histórica, las evidencias empíricas internacionales y en contexto peruano, para, finalmente, precisar las conclusiones y sugerencias a partir de las investigaciones desarrolladas en nuestro medio. Con esta información se evidencia la vigencia de un concepto que ha logrado aproximarse a diversos ámbitos educativos para comprender cómo orientar a los adolescentes y jóvenes hacia la consecución de sus metas personales.

Para ilustrar a través de un ejemplo el significado de la Perspectiva de Tiempo Futuro consideremos la siguiente situación:

Un/a adolescente de último año de secundaria aspira a ser profesional y estudiar en la universidad; pero piensa que no lo logrará porque la competencia en el examen de ingreso en una universidad pública es muy elevada; además, sus calificaciones no son altas y considera que su familia no tiene suficiente dinero para respaldarlo/a en una institución educativa privada; por tanto, de acuerdo a sus elaboraciones individuales, solo queda para él/ella, la opción de educación superior técnica. Ante ello es altamente probable que estas consideraciones guiarán su comportamiento presente. Sus temores tal vez lo/la detengan en la consecución de su meta y al tener miedo de fracasar evita dar el examen de admisión. No obstante, podría ocurrir algo totalmente distinto; si la esperanza de convertirse en profesional permite mantener su meta futura como una guía de comportamiento, probablemente se esforzará mucho para estar preparado y dar el examen de admisión en una universidad pública o privada (buscando opciones de beca y exoneración de pagos); a pesar de conocer que esto es difícil, hará lo posible por lograr esta meta; trabajará en lo que esté a su alcance para ahorrar dinero y utilizará distintas estrategias no solo para ingresar a la universidad, sino para mantenerse en ella y graduarse.

El ejemplo nos permite constatar que las metas futuras orientan nuestro comportamiento; pero, de acuerdo con nuestros temores o esperanzas, dirigimos nuestras acciones en el presente. Pero esta circunstancia no ocurre en todo ser humano, pues cada persona, de manera subjetiva, viaja a través del tiempo. Algunas personas eligen orientarse hacia acontecimientos del pasado y evocar de modo constante comportamientos o emociones del pasado; la famosa frase todo tiempo pasado fue mejor pone en evidencia este tipo de orientación temporal. Existe también la opción de centrarse en el presente y no proyectarse hacia metas que se distancien de las acciones vinculadas a circunstancias inmediatas. Pero otras personas, no obstante, orientan y precisan sus metas hacia el futuro, pues la mente humana posee esta posibilidad y por ello resulta viable anticipar lo que se puede lograr mediante planes y acciones organizadas (Andre, van Vianen, Peetsma \& Oort, 2018; Gilbert \& Wilson, 2007).

Considerando la extraordinaria condición humana de orientarse hacia el futuro, los investigadores han analizado, desde diversos ángulos teóricos, un constructo que evidencia la integración del futuro cronológico en la vida presente y cotidiana del ser humano (Lewin, 1942/1948; Lens, 1998). Se trata del concepto de Perspectiva de Tiempo Futuro (PTF) u Orientación Futura (OF) que, en su condición multidimensional, representa aquellas imágenes que consciente y subjetivamente elaboran las personas con relación al futuro (Seginer \& Lens, 2015; Seginer, 2009). Cabe señalar que ambas categorías varían levemente en su contenido, pero resaltan la condición cognitiva y motivacional de la variable y, por ello, en el presente artículo se aludirá a los dos términos como imágenes subjetivas con relación al futuro. Dichas imágenes se formulan como metas y permiten organizar planes diversos para alcanzarlas. Como consecuencia de esta posibilidad psicológica, centralmente motivacional, los comportamientos emitidos en el presente se convierten en submetas o acciones que instrumentalmente conducen a los individuos 
al logro de sus planes y metas futuras (Lens, et al., 2012; Locke \& Latham, 2004; Seginer \& Lens, 2015).

\section{Evolución histórica del concepto de perspectiva de tiempo futuro}

Para conocer la evolución del concepto resulta inevitable remitirse a sus orígenes y específicamente a tres autores que inicialmente aludieron la temática de Perspectiva de Tiempo; ellos son Israeli, Frank y Lewin. Estos tres académicos aportaron de modo notable al desarrollo del concepto y permitieron proponer su actual multidimensionalidad (Husman \& Lens, 1999; Seginer, 2009; Seginer \& Lens, 2015). Destaca, entre los teóricos mencionados, el aporte de Lewin quien conceptualizó la Perspectiva de Tiempo como la integración que el individuo tiene de su futuro y pasado psicológico en un momento dado. Este autor usó el término de futuro psicológico como imagen mental que impacta en las acciones y estado de ánimo de las personas en el presente. Con esta propuesta se introdujo la idea del poder motivacional del futuro psicológico, pues a través de esta condición era posible dirigir el comportamiento en el presente (Lewin, 1942; Cartwright, 1951; Seginer, 2009). Adicionalmente, la psicología científica de la época consideró al comportamiento como una acción intencionalmente orientada hacia una meta y dicha meta fue considerada como elaboración cognitiva con consecuencias motivacionales (Eysenck, 1952; Herrera \& Lens, 2009; Miller, Galanter \& Pribram, 1960; Weiner, 1992).

Tomando en consideración estos aportes, el concepto de tiempo psicológico fue calificado como representación mental y subjetiva que contiene, de modo integrado, imágenes del presente, pasado y futuro (Trommsdorff \& Lamm, 1975). Nuttin y Lens (1985) elaboraron de manera más profunda la propuesta sobre tiempo psicológico y remarcaron que la representación cognitiva del presente, pasado y futuro poseía una localización espaciotemporal en la mente de los individuos; dicha localización regularía el comportamiento de las personas en el presente. A partir de estas propuestas, surgieron ciertas conceptualizaciones sobre el pensamiento futuro entre las cuales destacan las siguientes formulaciones: se trata centralmente de la tendencia a orientarse hacia el futuro (Zimbardo \& Boyd, 1999); es un rasgo de personalidad (Gjesme, 1983; Zimbardo \& Boyd, 1999); es un constructo cognitivo-motivacional (Nuttin \& Lens, 1985; Seginer \& Lens, 2015); representa un concepto multidimensional (motivacional-cognitivo- comportamental (Seginer, et al., 1991); implica possible future selves (Markus \& Nurius, 1986). Cabe señalar que las diversas definiciones que reflejan el pensamiento futuro se encuentran conectadas al sustento teórico del cual emergen y por ello no son equivalentes.

Las múltiples conceptualizaciones elaboradas por distintos investigadores han sido organizadas en tres aproximaciones teóricas; específicamente Perspectiva de Tiempo Futuro (PTF); Orientación Futura (OF) y Possible selves (PS). La PTF es una aproximación motivacional que destaca la integración, en el presente psicológico, del futuro anticipado (De Volder \& Lens, 1982; Husman \& Lens, 1999; Lens, 1998; Nuttin \& Lens, 1985; Seginer \& Lens, 2015); claramente se asume que las imágenes elaboradas en relación a eventos futuros brindan sustento para establecer las metas futuras individuales. De modo similar, la Orientación Futura (OF) alude a imágenes que los seres humanos elaboran respecto a su futuro; dichas imágenes son representadas de modo consciente, son auto-reportadas y se encuentran articuladas a las esperanzas y temores que las personas poseen respecto a su futuro poniendo en evidencia la interacción de los tres componentes que son parte del modelo teórico, específicamente los componentes motivacional, cognitivo y comportamental (Seginer \& Lens, 2015; Trommsdorff \& Lamm, 1975). Finalmente, la aproximación de possible selves (PS) indica que las personas tienden a elaborar ideas respecto a aquello en lo que quisieran convertirse o también aquello que los atemoriza. Estas ideas incentivan el comportamiento futuro individual (Markus \& Nurius, 1986). Las mencionadas conceptualizaciones reflejan la diversidad teórica existente en la investigación vinculada al pensamiento futuro (Emmons, 1986; Strathman, Gleicher, Boninger \& Edwards, 1994; Snyder, et al., 1991). Cada una de estas aproximaciones sugiere diferentes instrumentos de medición y ello ha generado cierta controversia al aproximarse al concepto de PTF u OF. 
Seginer (2009) ha propuesto una clasificación de las diversas aproximaciones con relación al pensamiento futuro y ha organizado las propuestas teóricas de acuerdo con la perspectiva temática o atemática. La primera prioriza el contenido de las metas o imágenes que las personas elaboran con respecto al futuro considerando los dominios de vida que se encuentran relacionados con los contenidos priorizados. En el caso de los adolescentes o adultos jóvenes los dominios de vida son carrera/trabajo o familia/matrimonio. La segunda perspectiva, es decir la atemática, considera que las personas pueden presentar una tendencia generalizada a pensar en el futuro y anticiparlo, pero sin un claro contenido. Los estudios que se presentarán como evidencia empírica de la PTF y OF en contexto peruano se adscriben a la perspectiva temática.

\section{Evidencia empírica PTF/OF. Estudios desarrollados en Perú 1998-2018.}

En la presente revisión sistemática del concepto de PTF/OF en contextos educativos, se ha considerado argumentos teóricos y resultados de estudios empíricos desarrollados internacionalmente y en el Perú; para ello, se ha recurrido a fuentes publicadas en revistas científicas de psicología. El concepto de PTF tiene una larga historia, particularmente en Europa, en donde después de aproximadamente cincuenta años de investigación, se logró precisar, a partir de estudios empíricos, que las personas trasforman cognitivamente sus motivos y/o necesidades en metas motivacionales específicas, planes y proyectos. Las metas y los planes se articulan a un dominio de vida (en la adolescencia al dominio educativo/laboral o familiar) y sus contenidos se organizan en función al nivel evolutivo o de desarrollo de las personas (Lens, 1986; Nuttin \& Lens, 1985; Herrera \& Lens, 2009). Dichas metas se ubican temporalmente a lo largo de un continuo que se extiende desde metas a corto plazo hasta metas distantes. A mayor conocimiento sobre cuáles son las opciones viables o realistas de las metas futuras, mayor sustento para orientar el comportamiento hacia su cumplimiento (Cantor, Norem, Niedenthal, Langston, \& Brower, 1987; Husman \& Lens, 1999; Lens, 1986; Markus \& Nurius, 1987; Nurmi, 199; Trommsdorff, 1994).

Cabe señalar que, de acuerdo a teorías contemporáneas de la motivación (entre las que destacan la teoría de la autodeterminación), los comportamientos que se activan a partir de metas futuras son extrínsecamente motivados $y$, por tanto, podrían ser calificados como acciones generadas a partir de motivación de menor calidad (contraria a la elevada calidad motivacional implicada al existir motivación intrínseca); no obstante, resulta claro, a partir de la investigación empírica de los últimos años, que existen diferentes tipos de motivación extrínseca y cada una de ellas posee cualidades distintas (Deci \& Ryan, 2000; Lens, Paixao, Herrera \& Grobler, 2012; Ryan \& Deci, 2017). Por ello, resulta relevante analizar el contenido de las metas (intrínsecas vs extrínsecas), pues, pese a que hay distintos tipos de motivación extrínseca, las metas con contenidos intrínsecos crean motivación de más elevada calidad.

Una evidencia empírica de cómo el contenido de la meta afecta la calidad de la motivación fue presentada a través de un estudio experimental desarrollado en clases de educación física para estudiantes belgas de nivel intermedio. Cuando las metas eran planteadas en términos intrínsecos (actividad física como saludable) la calidad de la motivación era elevada; el esfuerzo y persistencia mayor e incluso se buscaba ser miembro activo de clubes deportivos. Ocurría lo contrario cuando las metas eran extrínsecas (actividad física centrada en la imagen o el atractivo) (Vansteenkiste, Simons, Soenens \& Lens, 2004). Otros estudios experimentales en el mismo contexto educativo belga reportaron resultados similares (Vansteenkiste, Matos, Lens $\&$ Soenens, 2007). Se desprende de estas investigaciones que para motivar a los estudiantes a aprender en las aulas y en el momento presente, resulta importante remitirse a metas futuras de contenido intrínseco (Vansteenkiste, Timmermans, Lens, Soenens \&Van den Broeck, 2008). 
Tomando en consideración los aportes de la ciencia psicológica y los estudios desarrollados en otros continentes, en Perú, la PTF ha sido medida y el contenido de esta analizada en niveles educativos distintos. Desde 1998 diversas investigaciones han sido implementadas y 2988 estudiantes participaron en las mismas. El primer estudio se aproximó al análisis de la inserción social de los estudiantes de secundaria en colegios públicos de Lima; dicha investigación fue longitudinal (1998-2002) y se usó como instrumento el Método de Inducción Motivacional para medir la PTF en tres momentos. (Herrera, 2002) Se inició la investigación con 321 participantes y se culminó con 174, de los cuales 163 aspiraban a formarse profesionalmente en la Universidad. Se pudo constatar, luego de realizar el análisis de contenido correspondiente, que hubo diferencias entre los dos niveles socioeconómicos analizados (bajo y medio). La frecuencia de respuestas que ponían en evidencia metas que expresaban auto-realización general fue más elevado en el nivel socio económico bajo (41\%) versus (33\%) en el nivel medio $\left(\chi^{2}(\mathrm{~N}=163)=27.27, \mathrm{p}<.00\right)$.

Este resultado no fue sorprendente, pues los participantes eran adolescentes de quinto de secundaria cuando se realizó la primera medición y era previsible que ellos/ellas reportaran las aspiraciones y deseos vinculados a auto-realización. Es importante señalar que los grupos de nivel socioeconómico bajo (10.71\%) y medio (14.85\%) que fueron evaluados reportaron también metas orientadas al dominio de vida educativa; ambos grupos presentaron respuestas orientadas a culminar una carrera y ser profesionales de éxito $\left(\chi^{2}(\mathrm{~N}=163)=10.71 ; \mathrm{p}<.00\right)$. No obstante, los del sector socioeconómico medio, a pesar de expresar un porcentaje más elevado de respuestas orientadas a ser profesionales de éxito, fueron quienes presentaron más respuestas que ponían en evidencia su deseo de culminar con éxito sus estudios de nivel secundaria y rendir el examen de admisión. Cabe señalar que los estudiantes en la mencionada investigación longitudinal no presentan respuestas muy frecuentes vinculadas a la educación técnica luego de culminar sus estudios escolares; tampoco expresan, con elevada frecuencia, metas vinculadas al empleo. A partir de las respuestas emitidas podría inferirse que los estudiantes de nivel más bajo poseen una PTF más larga que los de sector medio, pues ellos se proyectan a culminar exitosamente una carrera y no mencionan con la misma intensidad que los de sector medio, su deseo de acabar, con notas apropiadas, quinto de secundaria (Herrera, 2002; Herrera \& Lens, 2009).

En el estudio mencionado, al realizar tres mediciones durante los años 1998-2000, se pudo constatar que existía una brecha entre los planes educativos reportados al culminar la secundaria y las acciones emitidas por los adolescentes evaluados. La mayoría de los 174 estudiantes que participaron en la investigación en los treinta meses consecutivos, no rindieron el examen de admisión, es decir, no implementaron sus planes, explícitamente reportados, de convertirse en profesionales exitosos. Teóricamente las necesidades y motivos de los adolescentes (expresados a través de sus metas futuras), podrían traducirse a través de metas más específicas y realistas. Si deseaban culminar una carrera, tendrían que estudiar en una institución universitaria y para hacerlo el examen de ingreso es indispensable. La constatación empírica (a lo largo de dos años) del limitado número de universitarios (5\% de 174) inserto en estas instituciones, puso en evidencia que los adolescentes tenían planes poco realistas. Es altamente probable que, considerando la propuesta de Locke y Latham (2004), sus metas no fueron lo suficientemente específicas como para orientar los comportamientos requeridos para convertirse en un/a profesional exitoso/a y también es posible que dichas metas fueron de elevada complejidad y no totalmente auto establecidas. De acuerdo con lo constatado, para los estudiantes analizados, no fue importante culminar una carrera a pesar de haberlo declarado de modo explícito al finalizar sus estudios de nivel secundario (Herrera \& Lens, 2009).

Posteriormente, en cuatro estudios transversales realizados en Lima-Perú entre los años 2002-2006 se analizó el contenido de la PTF de adolescentes y jóvenes que se encontraban en diferentes niveles y tipos de institución educativa (colegios de nivel secundario; universidades, instituciones técnicas y academias preuniversitarias). Cada uno de estos cuatro estudios se centró en un tipo de institución educativa y se desarrollaron anualmente con el respaldo de la Universidad de Lima y la Universidad Católica de Lovaina (Herrera, 2009). Las metas de los 1608 
participantes fueron registradas, al igual que en el estudio longitudinal previamente mencionado, mediante el Método de Inducción Motivacional (Nuttin \& Lens, 1985). Se volvió a constatar que las metas expresadas con mayor frecuencia fueron las vinculadas a contenidos de auto-realización, ello era previsible por la edad de los participantes. Adicionalmente, se reportaron metas vinculadas al dominio de vida educativo y laboral. Estos datos también ratificaron lo esperado, pues los estudiantes evaluados se encontraban en entidades educativas que los preparaban para insertarse en otras instituciones formativas o los orientaban al mundo del trabajo. Cabe señalar que los estudiantes que ya se encontraban cursando estudios en la universidad fueron los que, comparativamente a los otros alumnos de las instituciones mencionadas, reportaron la mayor frecuencia de metas vinculadas a culminar los estudios universitarios de manera exitosa (19.6\%); no obstante, resulta relevante remarcar que en la muestra seleccionada, los alumnos de último año de secundaria presentaron sus aspiraciones de culminar una carrera exitosa, con un volumen de respuestas de $\left(8.22 \% ; \chi^{2}(3, \mathrm{~N}=1608)=1717.18 ; \mathrm{p}<.00\right)$. Los estudiantes que estaban registrados en las academias preuniversitarias, contrariamente y poniendo en evidencia diferencias significativas, solo reportaron el $7.25 \%$ de sus respuestas en esta categoría. No obstante, sus aspiraciones y deseos, elaborados y propuestos a través de metas futuras que indicaban que esperaban aprobar el examen de admisión fueron más altas que las de las otras instituciones, pues alcanzaron el $18.59 \%$ del total de sus repuestas. Cabe mencionar, que, en las otras instituciones estudiadas, específicamente en los centros educativos de nivel secundario, también se reportó explícitamente el deseo de aprobar el examen de admisión, pero el volumen de las respuestas en estos casos fue bajo; $6.83 \%$; en las universidades sumamente bajo $.01 \%$ y en las instituciones técnicas también $.18 \%\left(\chi^{2}(3, \mathrm{~N}=1608)=3454.88, \mathrm{p}<.00\right)$. Tal vez, pese a que las respuestas remitidas fueron de muy baja frecuencia, su existencia (es decir el reporte explícito de querer aprobar el examen de admisión) podría indicar que aún está presente en los estudiantes universitarios ( a pesar de encontrarse ya insertos en el estudio de una carrera) o en los alumnos de instituciones técnicas; el interés o aspiración de estudiar otra carrera, ya sea iniciando el proceso de admisión nuevamente y reingresando a la universidad o culminando los estudios técnicos y postulando posteriormente a la institución universitaria. Es relevante mencionar que, tal como se esperaba, los estudiantes de los institutos técnicos fueron los que reportaron mayor frecuencia de respuestas vinculadas a culminar exitosamente la carrera técnica (14.01\%) y conseguir empleo (5.47\%) (Herrera \& Lens, 2009; Lens, Paixao, Herrera \& Grobler, 2012).

Los hallazgos obtenidos mantuvieron vigente el interés científico de conocer las particularidades psicológicas de los adolescentes y jóvenes peruanos cuyas edades oscilan entre 14 y 29 años y que representan el $36.7 \%$ de la población (INEI, 2018). De acuerdo con la investigación empírica desarrollada se puede inferir que muchos adolescentes y jóvenes limeños no transforman sus metas motivacionales futuras en acciones. Muchos aspiran convertirse en profesionales exitosos, pero no implementan sus planes. Esta afirmación fue constatada a través de otro estudio, realizado también en Perú. Martinez (2009), describió la perspectiva temporal futura en un grupo de adolescentes de Lima y analizó las posibles diferencias entre la perspectiva temporal de adolescentes y jóvenes. Reportó que las metas de ambos grupos eran muy similares, pues se constató que no cambian de modo sustancial. Se indicó, a través de esta investigación que es en el período adulto donde se observan cambios notables; ello se articuló con los hallazgos de estudios previos en los cuales se analizó la Perspectiva Temporal Futura y Satisfacción con la vida a lo largo del ciclo vital (Martinez, 2004).

Con el propósito de contrastar esta información con datos reportados en otros países se realizaron nuevos estudios transversales con adolescentes y jóvenes de Costa Rica y USA. Las dos investigaciones registraron datos locales y los resultados fueron remitidos en el período (2007-2015). En los estudios transversales mencionados, se asoció la variable PTF (contenido y extensión) a riesgo (solo comparando Perú y Costa Rica en 411 alumnos/as de centros educativos privados y públicos) (Herrera, 2009); y a satisfacción con la vida (comparando Perú, Costa Rica y USA en 648 estudiantes). (Herrera, Matos, Martínez \& Lens, 2015) Al analizar las variables PTF y riesgo, se pudo constatar nuevamente que los 411 alumnos de centros educativos de nivel secundario reportaban un alto número de respuestas que aludían a autorrealización, pero, no eran 
categorías tan generales como las registradas en estudios anteriores. En el estudio se puso en evidencia que la autorrealización o "felicidad" se lograría a través de ciertos mecanismos específicos. Por ejemplo, "yo espero alcanzar la felicidad cuando tenga dinero suficiente para mantenerme" o "yo deseo intensamente ser feliz y lo lograré cuando sea profesional". Se constató, adicionalmente, que mujeres y hombres de ambos países (tanto de colegios privados como públicos) reportaban esta categoría con una frecuencia relativa que destacaba al ser comparada con el volumen de las categorías que reflejaban otras respuestas $(11.37 \%$ y $8.79 \%$ en hombres vs 10.49 y $10.37 \%$ en mujeres; $\left.\chi^{2}(3, \mathrm{~N}=411)=9.04, \mathrm{p}<.03\right)$. Al analizar las respuestas que representaban los deseos de convertirse en profesionales, eran las mujeres aquellas que reportaban un número más elevado de este tipo de respuestas $\left(7.71 \%\right.$ y $7.20 \%$ vs $6.71 \%$ y $3.83 \% ; \chi^{2}(3$, $\mathrm{N}=411)=32.87, \mathrm{p}<.00$ ). También fueron ellas las que reportaron una frecuencia más elevada de respuestas vinculadas al examen de admisión, pero el porcentaje de respuestas no fue elevado (3.90\%). La constatación empírica de que son las mujeres las que aspiran con mayor intensidad lograr la educación superior es altamente relevante, pues en ciertas ocasiones se señala, basándose en una elaboración estereotipada que las mujeres no siempre desean ir a espacios de educación superior.

Es importante señalar, adicionalmente, que al analizar la extensión de la PTF se observa que son muy pocas las respuestas que reportan metas futuras distantes; son las mujeres, tanto de colegio público como privado, las que presentan mayor frecuencia de respuestas en la categoría intermedia y son los hombres de colegio público aquellos que ponen en evidencia una PTF corta. Es altamente probable que la limitada situación socioeconómica de un grupo particular oriente a los estudiantes hacia metas muy inmediatas y que no se proyecten más allá del período escolar. No obstante, si las metas futuras guían el comportamiento, una opción de formación educativa (técnica o profesional) requiere metas más distantes y realistas. Varios análisis adicionales se realizaron para establecer cómo se asociaba la extensión de la PTF y la tendencia al riesgo reportada por los adolescentes. Se constató, a través de un ANOVA $(F=7.22, \mathrm{p}=0.01)$ diferencias significativas en la interacción país-tipo de colegio (privado/público) en términos de tendencia al riesgo de contraer enfermedades generadas por un inadecuado estilo de vida. Los estudiantes peruanos de colegios privados presentaban mayor tendencia al riesgo que los de Costa Rica; pero los alumnos de centros educativos públicos de Costa Rica presentaban mayor tendencia al riesgo que los peruanos del mismo tipo de centro (público). Adicionalmente, se pudo constatar que cuanto más larga es la PTF, menor es la tendencia al riesgo y viceversa (Herrera \& Lens, 2009).

La última investigación desarrollada en Perú con el método de inducción motivacional, pero en su versión revisada que incluía como dominio de vida el área del self además de los dominios de vida educativa y familiar (Herrera, Martinez, Lens, 2010; Herrera, Matos, Martinez \& Lens, 2015) comparó la PTF de jóvenes universitarios, peruanos, costarricenses y estadounidenses asociándola con bienestar subjetivo (satisfacción con la vida). La muestra seleccionada estuvo constituida por 648 estudiantes universitarios de tres países del continente americano: Perú $(\mathrm{n}=217)$; Costa Rica $(\mathrm{n}=202)$; y Estados Unidos $(\mathrm{n}=229)$. Se estudio la relación entre PTF (contenido de metas/extensión) y satisfacción con la vida. La razón de analizar la relación entre estas dos variables no solo estuvo sustentada en la relevancia motivacional de la PTF sino en la constatación empírica de su relación positiva de su longitud/extensión con resultados positivos. A mayor extensión se ha observado mayor compromiso escolar, manejo eficiente del tiempo como estrategia de aprendizaje; mayor persistencia, menor procrastinación y elevada satisfacción al estudiar (De Bilde, Vansteenkiste \& Lens, 2011; Harber, Zimbardo, \& Boyd, 2003; Horstmanshof \& Zimitat, 2007; Jackson, Fritch, Nagasaka, \& Pope, 2003; Peetsma, 1994; Zaleski, 1987). Los resultados del análisis de contenido y extensión de la PTF muestran que, al aplicar un ANOVA para observar si existen diferencias entre los países, fue evidente un efecto significativo $(F(2,642)=5.634 ; \mathrm{p}=.004)$. Los estudiantes peruanos mostraron una PTF más larga $(M=32.39 ; S D=11.20)$ que los de EE. UU. $(M=28.26 ; S D=11.21)$ y Costa Rica $(M=29.49 ; S D=11.49)$. Las respuestas vinculadas al contenido se jerarquizaron, en los tres países y se presentaron en el siguiente orden: educación (culminar exitosamente la carrera), trabajo y 
familia. Adicionalmente, hubo respuestas vinculadas al self, entre las cuales destacaban uso del tiempo libre y relaciones interpersonales. Estas categorías ratifican los hallazgos de estudios anteriores y nuevamente se puede inferir que su presencia está asociada al nivel de desarrollo de los participantes (la gran mayoría son adolescentes y jóvenes). Al continuar con el análisis se precisó la asociación entre las categorías (contenido de la PTF) y bienestar subjetivo (satisfacción con la vida). Se observa que la variable país y educación fueron predictores significativos de la satisfacción con la vida. La variable país explicó el $42.7 \%$ de la varianza. Al añadir la variable educación en la ecuación, solo agregó el $1.4 \%$ extra de la varianza. Por tanto, considerando que país era predictor de la satisfacción con la vida se aplicó un ANOVA para evaluar las diferencias entre los grupos evaluados. Los resultados de los análisis post hoc mostraron que Costa Rica tuvo la media más alta $(M=5.58)$ y Perú la más baja al evaluar satisfacción con la vida $(F(2,373)=$ $151.00, \mathrm{p}<.000)$.

La investigación ha continuado en el contexto peruano y desde el año 2016 hasta la actualidad, el modelo multidimensional de Orientación Futura se ha implementado. El instrumento propuesto por Seginer (2009) ha sido validado en muestras de estudiantes universitarios. La confiabilidad ha sido analizada y actualmente se cuenta con una prueba en condiciones psicométricas óptimas. Los últimos estudios realizados con estudiantes de psicología de universidades privadas indican que hay una estrecha relación entre expectativas como parte del componente motivacional $\left(r=.53^{* *}\right)$ y satisfacción con la vida. Con este nuevo sustento, la línea de investigación se aproxima a un espacio de análisis netamente cuantitativo que, a pesar de no distanciarse de la propuesta inicial de PTF que sustenta su análisis desde una perspectiva cognitivo motivacional, se apoya en una mirada multidimensional en donde el componente motivacional, cognitivo y comportamental están representados en un modelo de análisis distinto.

\section{Conclusiones y sugerencias}

La ciencia psicológica y la investigación en el ámbito motivacional se adscribe a la resolución aprobada por la Asamblea General de la ONU (2011) en la cual se precisa que la búsqueda de la felicidad representa un objetivo humano central y por tanto se convierte en una aspiración universal que requiere ser incluida en las políticas públicas de los países. Los estudios empíricos, realizados en grupos de adolescentes y jóvenes peruanos y de algunos países del continente americano, nos indican que convertirse en un profesional exitoso es uno de los deseos que explícitamente y de modo prioritario se ha planteado. No obstante, la proyección de las metas futuras distantes no es constante. La mayoría de los estudiantes proyecta sus metas a corto plazo. Dada la condición socioeconómica de los participantes evaluados (centralmente de nivel medio y bajo), tal vez la longitud de la PTF corta sea un recurso adaptativo. La constatación permanente de estos resultados permite realizar esta inferencia, pues es evidente que la categoría educación postsecundaria es relevante para el grupo de, aproximadamente, 3000 estudiantes analizados en los últimos 20 años. El esfuerzo de investigación realizada buscando articular la PTF, tendencia al riesgo y satisfacción con la vida ha reportado que el contenido de las metas y la extensión de la PTF están asociados con estas variables. No obstante, reorientar la propuesta conceptual hacia un modelo multidimensional sobre Orientación Futura puede brindar nuevas luces en el ámbito de la investigación psicológica motivacional.

La medición de la variable Orientación Futura ha evolucionado permitiendo su ampliación hacia un modelo multidimensional de tres componentes. Validar el instrumento propuesto por Seginer (2009) no descarta la opción de medir el constructo a través del Método de Inducción Motivacional (Nuttin \& Lens, 1985), pero reorienta la propuesta hacia un espacio netamente cuantitativo $\mathrm{y}$ articulando tres componentes (motivacional, cognitivo $\mathrm{y}$ comportamental). Esta variación permitirá articular propuestas de investigación que articulen diversas variables cuantitativas entre las que ocupe un lugar central la medición de la satisfacción con la vida y la felicidad. 
Se requiere investigaciones futuras que analicen la relación entre Orientación Futura, bienestar subjetivo o psicológico y otras variables del self, ello permitirá no solo describir y explicar el comportamiento sino predecirlo a favor de futuras intervenciones educativas en distintos momentos del ciclo vital que, al representar diferentes momentos evolutivos y de desarrollo, proponen también tareas de desarrollo distintas. De acuerdo con lo expuesto en el presente manuscrito, se considera de vital importancia profundizar en esta línea de investigación a fin de establecer nuevas inferencias científicas y propuestas claras de intervención en distintos contextos educativos.

\section{Referencias}

Andre, L., van Vianen, A. E. M., Peetsma, T. T. D., \& Oort, F. J. (2018). Motivational power of future time perspective: Meta-analyses in education, work, and health. PLOS ONE, 13(1), e0190492. doi: https://doi.org/ 10.1371/journal.pone.0190492

Cantor, N., Norem, J. K., Niedenthal, P.M., Langston, C. A., \& Brower, A. (1987). Life tasks and cognitive strategies in a life transition [Special issue]. Journal of Personality and Social Psychology: Person and Situation Interactions, 53, 1178-1191.

Cartwright, D. (1951). Lewin, Kurt. Field Theory of Social Science. Selected Theoretical Papers. New York: Harper \& Brothers.

De Bilde, J., Vansteenkiste, M., \& Lens, W. (2011). Understanding the association between future time perspective and selfregulated learning through the lens of self-determination theory. Learning and Instruction, 21(3), 332-344. doi: https://doi.org/10.1016/j.learninstruc.2010.03002

De Volder, M., \& Lens, W. (1982). Academic achievement and future time perspective as a cognitive-motivational concept. Journal of Personality and Social Psychology, 42(3), 566-571.

Deci, E. L., \& Ryan, R. M. (2000). The "what" and the "why" of goal pursuits: Human needs and the self-determination of behavior. Psychological Inquiry, 11(4), 227-268. doi: https://doi.org/10.1207/S15327965PLI1104_01

Eccles, J. S., \& Wigfield, A. (2002). Motivational beliefs, values, and goals. Annual Review of Psychology, 53(1), 109-132. doi: https://doi.org/10.1146/annurev.psych.53.100901. 135153

Emmons, R. A. (1986). Personal strivings: An approach to personality and subjective wellbeing. Journal of Personality and Social Psychology, 51(5), 1058-1068. doi: http://dx.doi.org/10.1037/0022-3514.51.5.1058

Eysenck, H. J. (1952). The scientific study of personality. London: Routledge \& Kegan Paul.

Gjesme, T. (1983). On the concept of future time orientation: Considerations of some functions' and measurements' implications. International Journal of Psychology, 18(1-4), 443-461.

Gilbert, D. T., \& Wilson, T. D. (2007). Prospection: Experiencing the Future. Science, 317(5843), 1351-1354. doi: https:// doi.org/10.1126/science.1144161

Harber, K. D., Zimbardo, P. G., \& Boyd, J. N. (2003). Participant self-selection biases as a function of individual differences in time perspective. Basic and Applied Social Psychology, 25(3), 255-264. doi: https://doi.org/10.1207/S15324834BASP2503_08

Herrera, D. (2002). Social Insertion of High School Graduates in Lima. A socio psychological study. Leuven: Katholieke Universitei Leuven.

Herrera, D., \& Lens W. (2009). Importancia de la Perspectiva Futura en el proceso de Inserción Social. En D. Herrera (Ed.) Teorías contemporáneas de la Motivación: Una Perspectiva Aplicada. (pp.295-320). Perú: Fondo Editorial PUCP.

Herrera, D., \& Matos, L. (2009). Desarrollo del concepto de motivación y su representación en distintas aproximaciones teóricas. En D. Herrera (Ed.) Teorías contemporáneas de la Motivación: Una Perspectiva Aplicada. (pp.17-37). Lima: Fondo Editorial PUCP.

Herrera, D. (2002). Social Insertion of High School Graduates in Lima. A socio psychological study. Leuven: Katholieke Universiteit Leuven.

Herrera, D., Martinez, P., \& Lens, W. (2010). Perspectiva de tiempo futuro ${ }^{\circledR}$. Informe final del proyecto de investigación: Proyecto: Perspectiva de Tiempo Futuro, estrés percibido, 
recursos de afrontamiento, patrones de perfeccionismo y satisfacción de vida en estudiantes universitarios de Perú, Costa Rica y Estados Unidos: Un estudio transcultural (Documento Inédito). Perú: PUCP.

Herrera, D.; Matos, L.; Martinez, P. \& Lens, W. (2015) Perspectiva de tiempo futuro y satisfacción con la vida en adolescentes: un estudio transcultural,(dedicado al Prof. Dr. Willy Lens). Revista de Orientación Educacional. 29 (55), 33-51

Horstmanshof, L., \& Zimitat, C. (2007). Future time orientation predicts academic engagement among first-year university students. British Journal of Educational Psychology, 77, 703718. doi: https://doi.org/10.1348/000709906X160778

Husman, J., \& Lens, W. (1999). The role of the future in student motivation. Educational Psychologist, 34, 113-125. doi: https://doi.org/10.1207/s15326985ep3402_4

INEI (2018). Perú, indicadores de ingreso y empleo por departamento.2007-2017. Perú: INEI.

Jackson, T., Fritch, A., Nagasaka, T., \& Pope, L. (2003). Procrastination and perceptions of past, present, and future. Individual Differences Research, 1(1), 17-28.

Lewin, K. (1942/1948). Time perspective and morale. In K. Lewin (Ed.). Resolving social conflict (pp. 103-124). New York, NY: Harper \& Brothers Publishers.

Lens, W. (1986). Future time perspective: A cognitive-motivational concept. In D. R. Brown \& J. Veroff, (Ed.), Frontiers of Motivational Psychology (pp. 173-190). New York, NY: Springer-Verlag.

Lens, W. (1998). El rol de la perspectiva futura en la motivación estudiantil. Persona 1, 67-91.

Lens, W., Paixao, M., Herrera, D., \& Grobler, A. (2012). Future time perspective as a motivational variable: Content and extension of future goals affect the quantity and quality of motivation. Japanese Psychological Research. 54(3), 321-33. doi: https://doi.org/10.1111/j.1468-5884.2012.00520.x

Locke, E., \& Latham, G. (2004). What Should We Do about Motivation Theory? Six Recommendations for the Twenty-First Century. The Academy of Management Review, 29(3), 388-403. doi: https://doi.org/10. 2307/20159050

Martinez, P. (2004). Perspectiva Temporal Futura y Satisfacción con la Vida a lo largo del Ciclo Vital (Tesis doctoral). Universidad Autónoma de Barcelona, España.

Martinez, P. (2009). Perspectiva Temporal Futura en la adolescencia tardía: transición a la adultez. En D. Herrera (Ed.) Teorías contemporáneas de la Motivación: Una Perspectiva Aplicada. (pp 321-336). Perú: Fondo Editorial PUCP.

Markus, H., \& Nurius, P. (1986). Possible selves. American Psychologist, 41, 954-969.

Markus, H., \& Nurius, P. (1987). Possible selves: The interface between motivation and the selfconcept. In K. Yardley \& T. Honess (Eds.), Self and identity: Psychosocial perspectives (pp. 157-172). Oxford, England: John Wiley \& Sons.

Miller, G. A., Galanter, E., \& Pribram, K. H. (1960). Plans and the structure of behavior. New York: Holt.

Nuttin, J., \& Lens, W. (1985). Future time perspective and motivation: Theory and research method. Leuven: University Press and Lauwrence Erlbaum Associates.

Nurmi, J. (1993). Adolescent development in an age graded context: the role of personal beliefs, goals and strategies in the tackling of developmental tasks and standards. International Journal of Behavioral Development, 16(2), 169-189.

Peetsma, T. (1994). Toekomstperspectief als voorspeller van inzet voor school (Future time perspective as a predictor of school engagement). Tijdschrift Voor Onderwijsonderzoek, 19(4), 331-342.

Reeve, J. (2005). Understanding motivation and emotion. Hoboken, New Jersey: Wiley.

Ryan, R. M., \& Deci, E. L. (2017). Self-determination theory: Basic psychological needs in motivation, development, and wellness. New York, NY, US: Guilford Press.

Seginer, R., \& Lens, W. (2015). Future orientation: different approaches, different cultures. En Stolarski, M., Fieulaine, N., \& VanBeek, W. (Eds.) Time Perspective Theory; review, research and application: Essays in honor of Philip G. Zimbardo. New York: Springer.

Seginer, R. (2009). Future orientation: Developmental and ecological perspectives. New York, US: Springer. 
Seginer, R., Nurmi, J. E., \& Poole, M. (1991). Adolescent future orientation in cross-cultural perspectives. Paper presented in the 11th meeting of International Society for the Study of Behavioral Development. Minneapolis, MN.

Strathman, A., Gleicher, F., Boninger, D. S., \& Edwards, C. S. (1994). The consideration of future consequences: Weighing immediate and distant outcomes of behavior. Journal of Personality and Social Psychology, 66, 742-752.

Trommsdorff, G. (1994). Future time perspective and control orientation: Social conditions and consequences. In Z. Zaleski (Ed.), Psychology of future orientation (pp. 39-62). Lublin, Poland: Towarzystwo Naukowe KUL.

Trommsdorff, G., \& Lamm, H. (1975). An analysis of future orientation and some of its social determinants. In Fraser, J. T., and Lawrence, N. (eds.) The Study of Time, II (pp. 343361). New York: Springer.

Vansteenkiste, M., Simons, J., Lens, W., Soenens, B., Matos, L., \& Lacante, M. (2004). Less is sometimes more: Goal-content matters. Journal of Educational Psychology, 96(4), 755764. doi: https://doi.org/10.1037/0022-0663.96.4.755

Vansteenkiste, M., Simons, J., Soenens, B., \& Lens, W. (2004). How to become a persevering exerciser? Providing a clear, future intrinsic goal in an autonomy supportive way. Journal of Sport and Exercise Psychology, 26(2), 232-249.

Vansteenkiste, M., Matos, L., Lens, W., \& Soenens, B. (2007). Understanding the impact of intrinsic versus extrinsic goal framing on exercise performance: The conflicting role of task and ego involvement. Psychology of Sport and Exercise, 8, 771-794. doi: https://doi.org/10.1016/j.psychsport.2006.04.006

Vansteenkiste, M., Timmermans, T., Lens, W., Soenens, B., \& Van den Broeck, A. (2008). Does extrinsic goal framing enhance extrinsic goal-oriented individuals' learning and performance? An experimental test of the match perspective versus self-determination theory. Journal of Educational Psychology, 100(2), 387-397. doi: http://dx.doi.org/10.1037/0022-0663.100.2.387

Weiner, B. (1992). Human motivation: Metaphors, theories, and research. Thousand Oaks, CA: Sage Publications.

Zaleski, Z. (1987). Behavioral effects of self-set goals for different time ranges. International Journal of Psychology, 22(1), 17-38

Zimbardo, P. G., \& Boyd, J. N. (1999). Putting time in perspective: A valid, reliable individual differences metric. Journal of Personality and Social Psychology, 77, 1271-1288. 